\title{
ON METRIC GRAPHS WITH PRESCRIBED GONALITY
}

\author{
FILIP COOLS AND JAN DRAISMA
}

\begin{abstract}
We prove that in the moduli space of genus- $g$ metric graphs the locus of graphs with gonality at most $d$ has the classical dimension

$$
\min \{3 g-3,2 g+2 d-5\} .
$$

This follows from a careful parameter count to establish the upper bound and a construction of sufficiently many graphs with gonality at most $d$ to establish the lower bound. Here, gonality is the minimal degree of a non-degenerate harmonic map to a tree that satisfies the Riemann-Hurwitz condition everywhere. Along the way, we establish a convenient combinatorial datum capturing such harmonic maps to trees.
\end{abstract}

\section{Definitions AND RESUlt}

Metric graphs. A topological graph is a topological space obtained by gluing a finite, disjoint union of closed intervals along an equivalence relation on the boundary points. If a topological graph $\Gamma$ is connected and the intervals from which it is glued are prescribed with a positive length, then $\Gamma$ becomes a compact metric space with shortest-path metric. Such a metric space is called a metric graph. The genus of a metric graph is its cycle space dimension. Here is a metric graph of genus 2 with edge lengths $a, b, c$ :

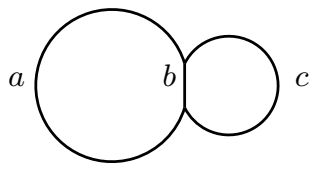

Every point $v$ in a metric graph has a neighbourhood isometric, for some positive $\epsilon$, to a finite union of half-open intervals $[0, \epsilon)$ glued (only) along 0. Each of these intervals is called a half-edge emanating from $v$, and their number is the valency of $v$. We identify two half-edges emanating from $v$ (for different $\epsilon$ ) if one is contained in the other.

Harmonic maps. A map $\varphi$ from a metric graph $\Gamma$ to a metric graph $\Sigma$ is called harmonic if it is continuous, linear with integral slopes outside a finite number of points, and if it moreover satisfies the following harmonicity condition at each point $v \in \Gamma$ : Fix a half-edge $e$ emanating from $w:=\varphi(v)$, and consider the sum of all slopes of $\varphi$ along half-edges emanating from $v$ that map to $e$. That sum, denoted $m_{\varphi}(v)$, should be independent of the choice of $e$. A harmonic map has a well-defined degree, defined as $\operatorname{deg} \varphi:=\sum_{v \in \Gamma, \varphi(v)=w} m_{\varphi}(v)$ for any $w \in \Sigma$. On the left is an example with $m_{\varphi}(v)=3$, and on the right is an example of a degree-2 harmonic map from our earlier genus-2 graph to a metric tree: 

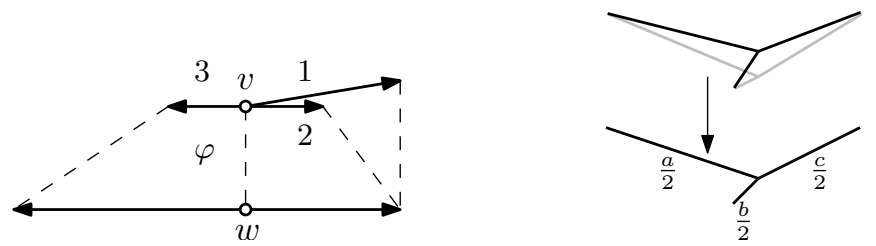

This definition is closely related to the notion of pseudo-harmonic indexed morphisms in Cap14 (which generalise harmonic morphisms from [BN09]) as follows: there the set-up concerns ordinary (non-metric) graphs, and the role of the index there is played by the slope in our definition. Moreover, in Cap14 the definition is extended to graphs in which the vertices are decorated with a non-negative genus; we do not do so here.

Riemann-Hurwitz conditions and tropical morphisms. Let $\varphi: \Gamma \rightarrow \Sigma$ be a harmonic map and $v \in \Gamma$. The Riemann-Hurwitz condition [BBM11, Definition2.2] on $\varphi$ at $v$ is the inequality

$$
k-2 \geq m_{\varphi}(v) \cdot(l-2),
$$

where $k$ is the valency of $v$ and $l$ the valency of $w:=\varphi(v)$. We will only be interested in harmonic maps that satisfy the Riemann-Hurwitz condition at all $v \in \Gamma$ and that, moreover, have non-zero integral slopes outside a finite number of points. This latter condition is equivalent to saying that $m_{\varphi}(v)$ is strictly positive at all $v \in \Gamma$. This is related to the condition of non-degeneracy in Cap14 as follows: there the non-degeneracy is required at vertices, and not at "internal points" of edges. But by folding each edge contracted to a point into two, one can create a harmonic morphism between corresponding metric graphs with non-zero slopes, albeit that the target graph needs to be modified as discussed below. The non-zero slope condition is also related to the modifiability condition in BBM14, Remark 2.5] and the finiteness condition in [ABBR15a, Definition 2.4].

We will call harmonic maps satisfying the Riemann-Hurwitz condition and the non-zero slope condition everywhere tropical morphisms between metric graphs.

Modifications. A modification of a metric graph $\Gamma$ is any metric graph $\Gamma^{\prime}$ obtained from $\Gamma$ by grafting a finite number of metric trees onto points of $\Gamma$. Given a modification $\Gamma^{\prime}$ of $\Gamma$ and a tropical morphism $\varphi: \Gamma \rightarrow \Sigma$, there exist a modification $\Gamma^{\prime \prime}$ of $\Gamma^{\prime}$, a modification $\Sigma^{\prime}$ of $\Sigma$, and a tropical morphism map $\varphi^{\prime}: \Gamma^{\prime \prime} \rightarrow \Sigma^{\prime}$ of the same degree as $\varphi$ and extending $\varphi$, constructed as follows. For any tree $S$ grafted onto $v \in \Gamma$ when going from $\Gamma$ to $\Gamma^{\prime}$, the graph $\Gamma^{\prime \prime}$ has $m_{\varphi}(v)-1$ additional copies of $S$ grafted onto $v$ and $m_{\varphi}\left(v^{\prime}\right)$ copies of $S$ grafted onto $v^{\prime}$ for each $v^{\prime} \in \varphi^{-1}(\varphi(v)) \backslash\{v\}$, and $\Sigma^{\prime}$ has a single copy of $S$ grafted onto $\varphi(v)$. Here we use that $m_{\varphi}(v)$ is positive, which follows from the fact that slopes are non-zero. The map $\varphi^{\prime}$ equals $\varphi$ on $\Gamma$ and maps the copies of $S$ in $\Gamma^{\prime \prime}$ to the single copy in $\Sigma^{\prime}$.

On the right is a modification of the harmonic morphism on the left:
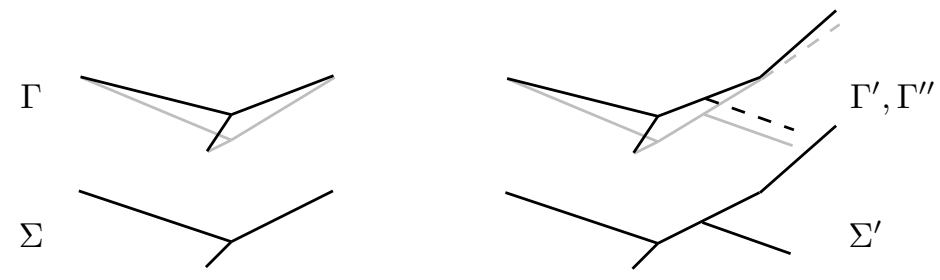
Here the two solid segments are grafted onto $\Gamma$ to arrive at $\Gamma^{\prime}$, and to arrive at $\Gamma^{\prime \prime}$ the two additional dashed lines are grafted.

Gonality. The tree gonality of a metric graph $\Gamma$ is the minimum degree of any tropical morphism from any modification $\Gamma^{\prime}$ of $\Gamma$ to a tree.

There are two notions of graph gonality in the literature, which are both inspired by the gonality of an algebraic curve. They are tree (or geometric) gonality and divisorial gonality. Each of these comes in several flavours, e.g. for ordinary or metric graphs Bak08, for graphs where the vertices can be decorated with higher genera Cap14, and for metrized complexes AB15, LM14. Yet another variant is stable gonality, which is the infimum of the divisorial gonality over all subdivisions of an ordinary graph CKK15. In this paper, gonality will always refer to tree gonality of metric graphs as defined above.

Given such a tropical morphism $\varphi: \Gamma^{\prime} \rightarrow T$ of degree $d$, and any $w \in T$, the divisor $D^{\prime}:=\sum_{v \in \varphi^{-1}(w)} m_{\varphi}(v) \cdot(v)$ on $\Gamma^{\prime}$ has degree $d$ and rank [BN07] at least one. Moving the chips of $D$ on grafted trees to their grafting points, one obtains a divisor $D$ on $\Gamma$ of degree $d$ and rank at least one. In particular, tree gonality is at least divisorial gonality. Divisorial gonality, in turn, is bounded from below by the treewidth of a graph or metric graph [vDdBG14, AK16].

The moduli space of metric graphs. Fix a natural number $g \geq 2$. For any genus- $g$ graph $G=(V, E)$ (that is, a connected graph with $|E|-|V|+1=g$; multiple edges and loops are allowed) with all valencies greater than or equal to 3 , we have $|E| \leq 3 g-3$, with equality if and only if all valencies are 3 . The positive orthant $M_{G}=\mathbb{R}_{>0}^{E}$ of edge lengths parameterises metric graphs of combinatorial type $G$. If $H$ is obtained from $G$ by contracting an edge $e$ (not a loop), then $H$ is another genus- $g$ graph, and we glue $M_{H}$ to $M_{G}$ as the coordinate hyperplane $\mathbb{R}_{>0}^{E \backslash\{e\}} \times\{0\}$. Similarly, if $H=\left(V^{\prime}, E^{\prime}\right)$ is a graph isomorphic to $G$ via an isomorphism $\sigma: E \rightarrow$ $E^{\prime}$, then we glue $M_{H}$ to $M_{G}$ via the induced bijection $\mathbb{R}_{>0}^{E^{\prime}} \rightarrow \mathbb{R}_{>0}^{E}$. In particular, we do this for automorphisms of $G$. This gluing of all $M_{G}$ where $G$ runs over all genus- $g$ graphs yields a topological space known as $\mathcal{M}_{g}^{\text {trop }}$, the moduli space of metric graphs of genus $g$. It has dimension $3 g-3$. By construction, every genus- $g$ graph $G=(V, E)$ defines a map $\mathbb{R}_{>0}^{E} \rightarrow \mathcal{M}_{g}^{\text {trop }}$. Every genus- $g$ metric graph is a modification of some graph represented by a point in $\mathcal{M}_{g}^{\text {trop }}$. The generalisation of $\mathcal{M}_{g}^{\text {trop }}$ to graphs with weighted vertices and marked points is discussed, for instance, in [Cap12, Section 3], and the topology of these spaces is the topic of [Koz09].

Main results. We will prove the following two theorems.

Theorem 1. For $d, g \geq 2$ the locus of metric graphs in $\mathcal{M}_{g}^{\text {trop }}$ that have gonality at most $d$ is closed of dimension $\min \{2 g+2 d-5,3 g-3\}$. In particular, the locus of genus-g metric graphs of gonality at least $\lceil(g+2) / 2\rceil$ is open and dense in $\mathcal{M}_{g}^{\text {trop }}$.

This theorem comprises two inequalities, and both will be proved by purely combinatorial means. Using the Kempf-Kleiman-Laksov existence result for special divisors Kem71, KL72] and ABBR15a, Corollary 3.25 and Corollary 4.28] (which are variants of Baker's specialisation lemma Bak08, Lemma 2.8] and of Conrad's result [Bak08, Corollary B.3], respectively), it follows that in fact all metric graphs in $\mathcal{M}_{g}^{\text {trop }}$ have gonality at most $\lceil(g+2) / 2\rceil$. We will give a constructive, purely combinatorial proof for the following statement. 
Theorem 2. Let $g \geq 1$ be a natural number. For any genus- $g$ graph $G=(V, E)$ all of whose vertices have valency 3 , the positive orthant $\left(\mathbb{R}_{>0}\right)^{E}$ contains a non-empty open cone $C_{G}$ whose image in $\mathcal{M}_{g}^{\text {trop }}$ consists entirely of metric graphs with gonality exactly $d:=\lceil(g+2) / 2\rceil$. Moreover, $C_{G}$ can be chosen such that every metric graph represented by a point in $C_{G} \cap \mathbb{Z}_{>0}^{E}$ has a degree-d divisor of rank 1 supported at integral points.

It has been conjectured that in fact all metric graphs with integral edge lengths admit such a divisor Bak08, Conjecture 3.10], but our methods do not imply that result. We further remark that by HKN13, on a metric graph with integral edge lengths, the rank of a divisor supported on the integral points equals its rank when regarded as a divisor on the natural ordinary graph whose vertices are the integral points (see also [Luo11]).

In [CKK15, Theorem B] an upper bound is established for the stable gonality of an ordinary graph, which is defined there as the infimum of the tree gonality over all subdivisions of the graph. Our Theorem 2 implies the special case of that theorem in which all vertices have valency at most three.

The fact that the locus of metric graphs of gonality (at least) $\lceil(g+2) / 2\rceil$ is open and dense in $\mathcal{M}_{g}^{\text {trop }}$ is an exact tropical analogue of the corresponding statement for algebraic curves. This is interesting as, so far, only the (divisorial) gonality of rather specific graphs (such as chains of loops) was well-understood CDPR12. Using the aforementioned variant of Baker's specialization lemma, our theorem implies that a general curve of genus $g$ has gonality at least $\lceil(g+2) / 2\rceil$ (the "non-existence part" of Brill-Noether theory). The idea that one does not need a specific graph to prove this statement but that, rather, a suitable dimension count suffices, goes back to a conference talk by Mikhalkin in 2011 [Mik11].

Remark 3. The dimension $2 g+2 d-5$ in Theorem 1 is equal to the dimension of the gonality- $d$ locus in the moduli space of genus- $g$ curves Seg28. Applying the tropicalisation map described in ACP15] to the latter locus might yield a different proof of the fact that the dimension in our theorem is at least $2 g+2 d-5$ : by the specialisation lemma, this map sends the classical gonality- $g$ locus into our gonality- $g$ locus, and its image should still have dimension $2 g+2 d-5$. On the other hand, ABBR15b, Theorem 5.4] shows an example of a genus-27 ordinary metric graph which, equipped with suitable edge lengths, has gonality 4 while it is not the tropicalisation of any 4-gonal curve of genus 27 . It seems very worthwhile to work out the details of the precise correspondence between the classical gonality- $d$ locus and the corresponding tropical locus - but our present goal to establish Theorems 1 and 2 using purely combinatorial means.

Our paper is organised as follows. In Section 2 we present a combinatorial datum that captures a metric graph together with a tropical morphism of degree $d$ to a tree. We call this a gluing datum. Using this datum, in Section 3 we prove the upper bound on the dimension of the locus of gonality- $d$ metric graphs in $\mathcal{M}_{g}^{\text {trop }}$. Finally, in Section 4 we construct the cone $C_{G}$ and variants of it for lower-than-maximal gonality, thus showing that the upper bound is in fact the right dimension.

The most important problem that we leave open is to find a combinatorial construction of a degree- $\lceil(g+2) / 2\rceil$ tropical morphism $\varphi$ from (a modification of) every metric graph $\Gamma$ of genus $g$ to a tree. Moreover, since our construction of such morphisms for graphs in the cone $C_{G}$ depends continuously on the edge lengths, 
and since the moduli space of metric graphs of genus $g$ is connected in codimension one by Cap12, it is natural to ask whether the space of such pairs $(\Gamma, \varphi)$ is in a suitable sense connected. Both of these problems are adressed in forthcoming work by the second author with Alejandro Vargas [DV17.

\section{The GLUing DATUM}

We define the following combinatorial gadget.

Definition 4. A gluing datum $(T, d, \sim)$ is a tuple consisting of a metric tree $T$, a natural number $d$, and an equivalence relation $\sim$ on the disjoint union $S:=$ $T_{1} \sqcup \cdots \sqcup T_{d}$ of $d$ copies of $T$ satisfying the following properties, in which $\psi_{i}: T \rightarrow T_{i}$ stands for the identification of $T$ with its $i$-th copy.

(1) If $v, w \in S$ satisfy $v \sim w$, then there exist $u \in T$ and $i, j \in[d]$ such that $\psi_{i}(u)=v \in T_{i}$ and $\psi_{j}(u)=w \in T_{j}$.

(2) For any pair $i, j$ the set $\left\{u \in T \mid \psi_{i}(u) \sim \psi_{j}(u)\right\}$ has finitely many connected components, each of which is closed.

(3) The topological graph $\Gamma$ obtained from $S$ by identifying points along the equivalence relation $\sim$ is connected.

(4) For every $w \in T$ and $i \in[d]$ we have the inequality

$$
\left(\sum_{e} k_{e}(w, i)\right)-2 \geq m(w, i)(l(w)-2)
$$

described further below.

We will elaborate on the last item further below. Here is an example with $d=3$ leading to a genus-3 graph; the colors are purely decorative. In the last picture, dangling trees have been removed.
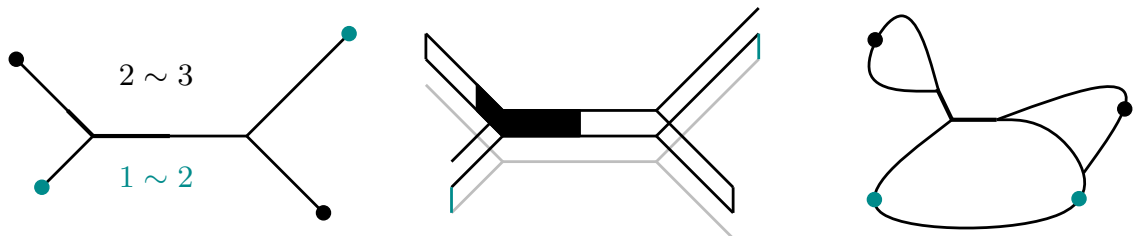

It is convenient to think of the $d$ copies of $T$ as lying above each other. Then the first condition says that a point is glued only to points vertically above or below it. The second condition says that gluing between two trees happens only along finitely many closed subsets. Given $u \in T$, if there exist $i, j \in[d]$ for which $u$ is a leaf of some tree in the forest in (2), then $u$ is called an endpoint of the gluing datum.

We turn the topological graph $\Gamma$ into a metric graph as follows: if for $v \in \Gamma$ the point $\varphi(v)$ is not an endpoint, then locally near $v$ a fixed number $d_{v}$ of the $T_{i}$ were glued together. Near $v$ we give $\Gamma$ the metric of $T$ divided by $d_{v}$, so that $\varphi: \Gamma \rightarrow T$ has slope equal to $d_{v}$ near $v$.

A gluing datum and a point $w \in T$ together give rise to an equivalence relation $\sim_{w}$ on the set $[d]$ defined by $i \sim_{w} j: \Leftrightarrow \psi_{i}(w) \sim \psi_{j}(w)$. By the closedness of the gluing point sets, the map $w \mapsto \sim_{w}$ is semicontinous in the following sense: for every $w \in T$ there is an open, connected neigbourhood $U$ of $w$ in $T$ such that $\sim_{u}$ is constant on each connected component of $U \backslash\{w\}$ and $\sim_{w}$ is a coarsening of each of these. 
The last condition in the definition can now be made explicit as follows. Given $w \in T$ and $i \in[d]$, let $m(w, i)$ be the cardinality of the equivalence class of $i$ in $\sim_{w}$ and let $l(w)$ be the valency of $w$ in $T$. For each half-edge $e \subseteq U$ emanating from $w$ let $k_{e}(w, i)$ be the number of equivalence classes into which the $\sim_{w}$-class of $i$ splits in the refinement $\sim_{u}$ for $u \in e \backslash\{w\}$. Then

$$
\left(\sum_{e} k_{e}(w, i)\right)-2 \geq m(w, i) \cdot(l(w)-2),
$$

where the sum is over all half-edges in $T$ emanating from $w$.

Proposition 5. Let $(T, d, \sim)$ be a gluing datum, and let $\Gamma=S / \sim$ be the topological space obtained from it, equipped with the aforementioned metric. Then the natural map $\varphi: \Gamma \rightarrow T$ is a tropical morphism of degree $d$.

Proof. The map $\varphi$ is continuous; this follows from the universal property of the quotient map $S \rightarrow \Gamma=S / \sim$, namely, that any map from $S$ into a topological space such that points equivalent under $\sim$ are mapped to the same point (here the map $S \rightarrow T$ ) factorises through a continuous map from $\Gamma$ (here $\varphi$ ). The slope of $\varphi$ along any half-edge emanating from a point $v$ that is not an endpoint is $d_{v}>0$.

To see that $\varphi$ is harmonic, let $v \in \Gamma$ and let $e$ be a half-edge emanating from $w:=\varphi(v)$ in $T$. Then the points near (but unequal to) $w$ along $e$ induce a fixed equivalence relation $\sim_{e}$ on $[d]$, which refines the equivalence relation $\sim_{w}$ on $[d]$. Let $I \subseteq[d]$ be the equivalence class of $\sim_{w}$ consisting of those $i$ for which $v$ is the image of $\psi_{i}(w)$. This class decomposes as a disjoint union $I_{1} \sqcup \cdots \sqcup I_{k}$ of equivalence classes under $\sim_{e}$. The latter classes correspond bijectively to the half-edges of $\Gamma$ emanating from $v$ that map to $e$. On the half-edge corresponding to $I_{j}$, the metric has been defined such that the slope of $\varphi$ equals $\left|I_{j}\right|$. Adding up all these slopes yields $m_{\varphi}(v):=|I|$, which is an invariant of $v$ and in particular independent of the half-edge $e$. This proves harmonicity. The degree is $d$ because that is the sum of the cardinalities of all equivalence classes of $\sim_{w}$.

Finally, to establish the Riemann-Hurwitz conditions for $\varphi$ at the the image $v$ in $\Gamma$ of $\psi_{i}(w)$, we note that $m(w, i)$ is the sum of all the slopes of $\varphi$ along each edge $e$ emanating from $w$ and that $\sum_{e} k_{e}(w, i)$ is the valency of $v$ in $\Gamma$. Hence the inequality (4) is precisely the Riemann-Hurwitz condition.

As we will prove next, every tropical morphism to a tree arises from a suitable gluing datum. This datum is not unique. First, there is the obvious ambiguity arising from permuting the copies $T_{1}, \ldots, T_{d}$. But in fact there is more ambiguity, as will become apparent in the following proof.

Proposition 6. Let $\varphi: \Gamma \rightarrow T$ be a tropical morphism. Then there exists a gluing datum $(T, d, \sim)$ that gives rise to $\varphi$.

Proof. The number $d$ is defined as the degree of $\varphi$. Without loss of generality, we may assume that the pre-image of some leaf $w_{0}$ of $T$ consists of $d$ distinct leaves $v_{1}, \ldots, v_{d}$ of $\Gamma$ with $m_{\varphi}\left(v_{i}\right)=1$ for all $i$. This situation can be achieved by grafting an additional interval on some leaf of $T$ and extending the morphism as discussed in the section on modifications.

We now construct the gluing relation $\sim$ on the disjoint union $T_{1} \sqcup \cdots \sqcup T_{d}$ of $T$, by determining at points $w \in T$ the local relation $\sim_{w}$, or equivalently, the partition $\Pi_{w}$ of $[d]$ into the equivalence classes of $\sim_{w}$. 
We will do this by walking along $T$ and starting at $w_{0}$. For the gluing relation $\sim_{w_{0}}$ at the leaf $w_{0} \in T$, choose for $\Pi_{w_{0}}$ the partition $\Pi_{1}$ of $[d]$ into singletons. Now we begin to move $w$ along $T$, starting at $w_{0}$ and tracing the pre-image $\left\{v_{1}, \ldots, v_{d}\right\}$ under $\varphi$ along. Let $w^{\prime} \in T$ be a point above which some of the $v_{i}$ converge. For all points $w$ on $T$ in between $w_{0}$ and $w^{\prime}$, we keep the partition $\Pi_{w}=\Pi_{1}$. However, above $w^{\prime} \in T$, we are forced to mimic the convergence in the gluing datum by making $\Pi_{w^{\prime}}$ the corresponding coarsening of $\Pi_{1}$. Let $e_{1}, \ldots, e_{l}$ be the half-edges emanating from $w^{\prime}$ in $T$, where $e_{1}$ is in the direction of $w_{0}$. Along the remaining half-edges $e_{2}, \ldots, e_{l}$ emanating from $w^{\prime}$ (so all except for $e_{1}$ ), we still have to choose the partitions $\Pi_{2}, \ldots, \Pi_{l}$ of $[d]$. They should satisfy the following rules. First, each $\Pi_{i}$ should be a refinement of $\Pi_{w^{\prime}}$. Second, the number of parts of $\Pi_{i}$ into which the part of $\Pi_{w^{\prime}}$ corresponding to a $v \in \varphi^{-1}\left(w^{\prime}\right)$ splits equals the number of half-edges in $\Gamma$ emanating from $v$ that map to $e_{i}$. Third, the cardinalities of these parts should equal the slopes of $\varphi$ along those half-edges. As $\varphi$ is harmonic, these slopes add up to the same number $m_{\varphi}(v)$ for all $i$, so that such partitions $\Pi_{2}, \ldots, \Pi_{l}$ certainly exist. Now fix any partitions $\Pi_{2}, \ldots, \Pi_{l}$ that satisfy the above rules. Analogously, we can continue moving the point $w \in T$ and define the partition $\Pi_{w}$ until we have done this for all $w \in T$. The corresponding gluing datum $(T, d, \sim)$ gives rise to the map $\varphi$ and satisfies the inequality (4) since $\varphi$ satisfies the Riemann-Hurwitz conditions.

One can make use of the ambiguity in the construction of the gluing datum $(T, d, \sim)$ (in particular, in the particular choice of the partitions $\left.\Pi_{2}, \ldots, \Pi_{l}\right)$ to impose convenient extra conditions on the gluing datum, for instance as follows:

Lemma 7. Any tropical morphism $\varphi: \Gamma \rightarrow T$ arises from a gluing datum $(T, d, \sim)$ in which for each $i \neq j$ in $[d]$ the set $\left\{w \in T \mid i \sim_{w} j\right\}$ is homeomorphic to a disjoint union of closed intervals.

The closed intervals in the lemma are allowed to consist of a single point.

Remark 8. Originally, we believed that the condition that any two trees are glued along intervals also implies the Riemann-Hurwitz conditions. However, as pointed out by Alejandro Vargas, this is incorrect. The following shows a piece of a gluing datum that satisfies the condition of the lemma but does not satisfy the RiemannHurwitz condition at the central point:

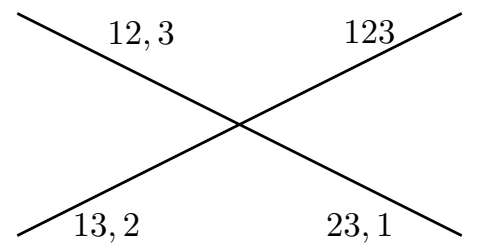

Here 12,3 is short-hand for the partition $\{\{1,2\},\{3\}\}$, etc. So the valency of the metric graph is $k=7$ at this vertex, that of the tree is $l=4, m_{\varphi}$ equals 3 but $k-2 \nsupseteq 3 \cdot(l-2)$.

Proof of Lemma 7 . The condition in the lemma translates to the following combinatorial condition on the partitions $\Pi_{1}, \ldots, \Pi_{l}$ along the half-edges emanating from a point $w \in T:$ if $i, j$ are distinct elements in the same part of some partition $\Pi_{h}$, then there is at most one $h^{\prime} \neq h$ such that $i, j$ are in the same part of $\Pi_{h^{\prime}}$. Lemma 9 
below shows that, when $\Pi_{1}$ is prescribed, the Riemann-Hurwitz condition implies that $\Pi_{2}, \ldots, \Pi_{l}$ can be chosen to ensure this.

Lemma 9. Let $l, m, k_{1}, \ldots, k_{l}$ be positive integers and assume that $k_{1}+\cdots+k_{l}-2 \geq$ $m(l-2)$. For each $h=1, \ldots, l$ let $\pi_{h}$ be a partition of the number $m$ with $k_{h}$ (nonzero) parts; in particular, each $k_{h}$ is at most $m$.

Then there exist partitions $\Pi_{1}, \ldots, \Pi_{l}$ of the set $[m]$ such that $\pi_{h}$ records the sizes of the parts in $\Pi_{h}$ and such that the coarsest common refinement of any three of the $\Pi_{h}$ is the partition into singletons.

We dub this property of a sequence of partitions the triple intersection property.

Proof. We proceed by induction on $m$. The statement is trivially true for $m=1$ : the only choice for each $\Pi_{h}$ is the partition of [1] into the one singleton $\{1\}$, and this choice satisfies the triple intersection property. Now let $m \geq 2$, assume that the statement is true for $m-1$, and consider $k_{1}, \ldots, k_{l}$ and partitions $\pi_{h}$ of $m$ as in the lemma. Order the $k_{h}$ such that $k_{1} \leq \ldots \leq k_{l}$.

If $k_{2}=m$, then the only choices for $\Pi_{2}, \ldots, \Pi_{l}$ are the partitions into singletons, and any choice for $\Pi_{1}$ will do. Hence we may assume that $k_{1}, k_{2}<m$, so that $\pi_{1}, \pi_{2}$ contain parts $a_{1}, a_{2}>1$, respectively. Next, we have $k_{3}>1$ because otherwise

$$
\sum_{h} k_{h}-2 \leq 3 \cdot 1+(l-3) \cdot m-2<(l-2) m,
$$

where we use that $m>1$. Hence $\pi_{3}$ contains at least two parts $a_{3}, b_{3}>0$. Similarly, we find that $k_{4}>m / 2$, hence each $\pi_{h}$ with $h \geq 4$ contains at least one 1 .

Now construct $\pi_{h}^{\prime}$ from $\pi_{h}$ as follows: for $h=1,2$ reduce the part $a_{h}$ by 1 ; for $h=3$ replace the two parts $a_{3}, b_{3}$ by a single part $a_{3}+b_{3}-1$; and for $h \geq 4$ discard a part equal to 1 . This yields $l$ partitions of $m-1$, and in the inequality of the lemma both sides have been reduced by $l-2$. By the induction hypothesis, there are partitions $\Pi_{h}^{\prime}, h=1, \ldots, l$ of $[m-1]$ corresponding to $\pi_{h}^{\prime}$ that satisfy the triple intersection property.

From the $\Pi_{h}^{\prime}$ we construct partitions $\Pi_{h}$ of $[\mathrm{m}]$ as follows. For $h=1,2$ let $A_{h}^{\prime}$ be the (or a) part of $\Pi_{h}^{\prime}$ of cardinality $a_{h}-1$; add $m$ to this set to obtain $A_{h}$. For $h>3$ add the singleton $\{m\}$ to the partition $\Pi_{h}^{\prime}$. Finally, for $h=3$ let $A_{3}^{\prime}$ be the part of $\Pi_{3}^{\prime}$ of size $a_{3}+b_{3}-1$. We want to replace $A_{3}^{\prime}$ with two sets $B_{3}$ and $A_{3}:=\left(A_{3}^{\prime} \backslash B_{3}\right) \cup\{m\}$ where $B_{3} \subseteq A_{3}^{\prime}$ has cardinality $b_{3}$. The only triple intersection that might now get cardinality 2 is the one between $A_{1}, A_{2}, A_{3}$ (which contains $m$ ), but this happens only if we put an element of $A_{1}^{\prime} \cap A_{2}^{\prime} \cap A_{3}^{\prime}$ in $A_{3}$. Since this intersection contains at most one element, we can avoid this by putting that element, if it exists, into $B_{3}$ (whose prescribed cardinality $b_{3}$ is positive).

Remark 10. We think that a generalisation of Lemma 9 might hold, where one replaces 2 by an $n \in\{0, \ldots, l-1\}$, the inequality by $k_{1}+\cdots+k_{l}-n \geq m(l-n)$, and the triple intersection property by the property that the coarsest common refinement of any $n+1$ of the partitions be the partition into singletons. But since we do not need this for our current purposes, we have not pursued this.

Remark 11. Lemma 7 will not be used in the remainder of the paper. It may, however, prove useful when actually generating many points in the gonality- $d$ locus of $\mathcal{M}_{g}^{\text {trop }}$. 
Now that we know that gluing datums give rise to tropical morphisms and vice versa, we can express the genus of a metric graph $\Gamma$ in terms of a gluing datum as follows.

Proposition 12. Let $(T, d, \sim)$ be a gluing datum. For each subset $I \subseteq[d]$ define

$$
T_{I}:=\left\{w \in T \mid \forall i, j \in I: i \sim_{w} j\right\} \subseteq T .
$$

Then the genus of the metric graph $\Gamma$ determined by the datum equals

$$
g(\Gamma)=\sum_{I \subseteq[d]}(-1)^{|I|} c\left(T_{I}\right)
$$

where $c\left(T_{I}\right)$ is the number of connected components of $T_{I}$.

In this proposition, $T_{I}$ is the image in $T$ of the intersection of the images in $\Gamma$ of the $T_{i}$ with $i \in I$, and a closed forest in $T$. Note that $T_{I}$ is equal to $T$ for $|I|<2$.

Proof. The genus of $\Gamma$ equals 1 minus its Euler characteristic. Since the Euler characteristic of a forest is its number of connected components, the formula follows immediately from the usual inclusion-exclusion formula for the Euler characteristic of simplicial complexes - the additional term 1 corresponds to the term with $I=\emptyset$ on the right-hand side.

\section{UPPER BOUNDS ON THE DIMENSION OF THE LOCUS OF BOUNDED GONALITY}

Our goal in this section is to derive the upper bound from Theorem 1 on the dimension of the locus in $\mathcal{M}_{g}^{\text {trop }}$ where the gonality is equal to $d$. For a gluing datum $(T, d, \sim)$ recall that $w \in T$ is called an endpoint if it is a leaf of some connected component of $T_{\{i, j\}}$ for some distinct $i, j \in[d]$; and we write $E$ for the set of endpoints that have valency at most 2 in $T$. We also introduce the following notation: if $\Gamma$ is the corresponding metric graph and $\varphi: \Gamma \rightarrow T$ the tropical morphism defined by the datum and $v \in \Gamma$ a point with valency $k$ where $\varphi(v)$ has valency $l$ in $T$, then we set $r_{\varphi}(v):=(k-2)-m_{\varphi}(v)(l-2)$. By the Riemann-Hurwitz condition, this is a nonnegative number. Note that $r_{\varphi}(v)$ is positive only at a finite number of points.

Proposition 13. Let $(T, d, \sim)$ be a gluing datum, $\varphi: \Gamma \rightarrow T$ the corresponding tropical morphism, and $g$ the genus of $\Gamma$. Then we have

$$
|E|+\sum_{v \in \Gamma: \varphi(v)} r_{\text {has valency }>2} r_{\varphi}(v) \leq 2 g+2 d-2 .
$$

Proof. Since $\Gamma$ is connected by assumption, we may order the copies of $T$ such that each $T_{i}$ with $i>1$ is glued to at least one $T_{j}$ with $j<i$. For each $e \in[d]$, the restriction of $\sim$ to $T_{1} \sqcup \ldots \sqcup T_{e}$ then yields a gluing datum $(T, e, \sim)$ of a connected metric graph $\Gamma_{e}$, which is obtained from $\Gamma_{e-1}$ by suitably gluing the copy $T_{e}$ to it.

We argue by induction on $e$. For $e=1$, the statement is true: $E=\emptyset, r_{\varphi}(v)=0$ for all $v$, and $0+0=2 \cdot 0+2 \cdot 1-2$. For the induction step, assume that the formula holds for $(T, e-1, \sim)$, and consider the graph $\Gamma_{e}$. Let $C \subset \Gamma$ be the set along which $T_{e}$ is glued to $\Gamma_{e-1}$. The increase in $g$ when passing from $\Gamma_{e-1}$ to $\Gamma_{e}$ equals $c(C)-1$, and the increase in $d$ is 1 . Hence the right-hand side in 1 increases with $2 c(C)$.

On the other hand, consider a connected component $C^{\prime}$ of $C$ and let $v \in C^{\prime}$. Denote by $l$ the valency of $\varphi(v)$ in $T$ and by $k_{v}^{\prime}$ the valency of $v$ in the closed tree 
$C^{\prime} \subset \Gamma$. Then $m_{\varphi}(v)$ increases by 1 in passing from $\Gamma_{e-1}$ to $\Gamma_{e}$, while the valency $k$ of $v$ in these graphs increases by $l-k_{v}^{\prime}$. This means that $r_{\varphi}(v)$ increases by $l-k_{v}^{\prime}-(l-2)=2-k_{v}^{\prime}$ (so a decrease if $k_{v}^{\prime}>2$ ).

If $C^{\prime}$ consists of the single point $v$ only, then $v$ either contributes to an increase of $|E|$ by at most 1 (if $l \leq 2$ ) or to an increase by $2-k_{v}^{\prime}=2$ of the second summand in the left-hand side of (1) (if $l>2$ ). In either case, $C^{\prime}$ contributes an increase of at most 2 to the left-hand side.

Now suppose that $C^{\prime}$ is not a single point. Then each leaf $v$ of $C^{\prime}$ has $k_{v}^{\prime}=1$ and contributes at most 1 to the left-hand side: to $|E|$ if $l \leq 2$ or to the other summand if $l>2$. A $v \in C^{\prime}$ which is not a leaf cannot be a new point in $E$. Indeed, if it is a leaf of a connected component of $T_{\{i, e\}}$ with $i \neq e$ and also has valency at most two in $T$, then it has valency exactly two, and any half-edge emanating from $v$ not contained in $T_{\{i, e\}}$ (of which there are one or two) must be contained in some $T_{\{j, e\}}$ with $j \neq i, e$. But then $v$ is a leaf of the component of $T_{\{i, j\}}$ containing $v$, hence already in $E$ and not a new endpoint. Hence $C^{\prime}$ contributes an increase of the left-hand side in (1) by at most

$$
\#\left\{\text { leaves of } C^{\prime}\right\}+\sum_{v \text { non-leaf in } C^{\prime}}\left(2-k_{v}^{\prime}\right) \text {. }
$$

Straightforward combinatorics shows that this quantity equals 2 for every tree that is not a single point.

Since each component of $C$ contributes at most 2, the left-hand side of (1) increases by at most $2 c(C)$ in passing from $\Gamma_{e-1}$ to $\Gamma_{e}$, and combining this with the first paragraph of the proof we find that the inequality is preserved.

Corollary 14. In the setting of Proposition 13 we have $|E| \leq 2 g+2 d-2$.

Proof. This follows immediately from the Riemann-Hurwitz condition that $r_{\varphi}(v) \geq$ 0 for each $v$.

We can now prove that the dimension of the locus in $\mathcal{M}_{g}^{\text {trop }}$ where the gonality is at most $d$ is at most $2 g+2 d-5$.

Proof of Theorem 1, upper bound. Start by taking a gluing datum $(T, d, \sim)$ that gives rise to a degree- $d$ tropical morphism $\varphi: \Gamma \rightarrow T$, and assume that $\Gamma$ has genus $g$. If $v$ is a leaf of $T$ such that $\sim_{v}$ is the partition of $[d]$ into singletons, then $\varphi^{-1}(v)$ consists of $d$ valency-one points. If the interval $e$ leading to $v$ contains points where the gluing is not trivial, then let $w \in e$ the point closest to $v$ with this property. Otherwise, let $w \in T$ be the point of valency greater than two where $e$ is attached to the rest of $T$. By deleting the segment $(w, v]$ we obtain a new gluing datum $\left(T^{\prime}, d, \sim\right)$ defining a metric graph $\Gamma^{\prime}$ of which $\Gamma$ is a modification. Proceeding in this manner with deleting unnecessary leaves, we arrive at a gluing datum, which we still denote $(T, d, \sim)$, such that $\sim_{v}$ is non-trivial at every leaf $v \in T$. This means that all leaves are endpoints. Next re-attach an interval of positive length at each leaf $v$ where the gluing relation is constant and equal to $\sim_{v}$. These intervals do not contribute to the edge lengths of $\Gamma$ accounted for in the moduli space, and we introduce them merely because they $d o$ contribute to the parameter count that follows. We denote the resulting gluing datum again by $(T, d, \sim)$.

Now we count the cardinality $|V \cup E|$ where $V \subseteq T$ is the set of points of valency greater than 2 and $E$ is the set of endpoints of valency at most 2. By basic combinatorics, $|V|$ is at most $l-2$ where $l$ is the number of leaves, with 
equality if and only if all points in $V$ are trivalent. Thus, by the corollary above, $|V \cup E| \leq 2 g+2 d-4+l$. Since all leaves of $T$ are end points, the set $V \cup E$ can be seen as a vertex set of $T$. Hence, the complement of $V \cup E$ in $T$ consists of at most $2 g+2 d-5+l$ intervals whose lengths determine the lengths in $\Gamma$. Among these intervals, $l$ intervals at the leaves do not contribute to the image of $\Gamma$ in the moduli space (these intervals may be longer than the ones added in the last modification, if the gluing relation was already constant on a postive-length interval leading into a leaf $v$ ). Thus we arrive at the correct dimension count $2 g+2 d-5$.

To describe the locus in $\mathcal{M}_{g}^{\text {trop }}$ where the gonality is at most $d$ one now proceeds as follows:

(1) Enumerate all (finite, combinatorial) trees with at most $2 g+2 d-5$ edges (whose vertices are allowed to have any valency greater than or equal to 1 ); there are finitely many of these.

(2) Equip each such tree with a gluing relation $\sim$ that is constant along the edges; again, there are finitely many possibilities.

(3) Select those combinatorial choices of a tree $T$ plus a gluing that lead to a connected graph $\Gamma$ of genus $g$ and a map $\Gamma \rightarrow T$ that satisfies the RiemannHurwitz conditions. (In practice, one might want to exploit Lemma 7 in the previous step to rule out some of the possibilities.)

(4) Writing $E$ for the edge set of $T$, we obtain a map $\Psi$ from $\mathbb{R}_{\geq 0}^{E}$ into $\bigsqcup_{g^{\prime} \leq g} \mathcal{M}_{g^{\prime}}^{\text {trop }}$ by interpreting the entries of $\ell \in \mathbb{R}_{\geq 0}^{E}$ as edge lengths on $T$-we take the union over all $g^{\prime} \leq g$ since cycles get contracted if their total length is zero. The set $\Psi\left(\Psi^{-1}\left(\mathcal{M}_{g}^{\text {trop }}\right)\right)$ is a closed cell in $\mathcal{M}_{g}^{\text {trop }}$ of dimension at most $2 g+2 d-5$.

The union of these finitely many cells is the locus in the theorem.

\section{Constructing graphs With PREscribed gonality}

In this section we construct a subset of $\mathcal{M}_{g}^{\text {trop }}$ of dimension $\min \{2 g+2 d-5,3 g-3\}$ where the gonality is at most $d$, thus proving the lower bound in Theorem 1 . Consequently, we prove that for $d=\left\lceil\frac{g}{2}\right\rceil+1$ this subset intersects each cell in the definition of $\mathcal{M}_{g}^{\text {trop }}$ in a non-empty open subset. The main construction is given in the following subsection.

Gluing in a tripod. Let $(T, d, \sim)$ be a gluing datum with corresponding tropical morphism $\varphi: \Gamma \rightarrow T$. Pick points $u, v, w \in \Gamma$ with images $u^{\prime}:=\varphi(u), v^{\prime}:=$ $\varphi(v), w^{\prime}:=\varphi(w) \in T$. These points span a tree in $T$ with at most three leaves; let $y$ be the unique point in $T$ that lies on all the shortest paths $u^{\prime} v^{\prime}, u^{\prime} w^{\prime}$, and $v^{\prime} w^{\prime}$. Let $a, b, c$ be the lengths of the shortest paths $u^{\prime} y, v^{\prime} y, w^{\prime} y$, respectively, and pick additional lengths $a^{\prime}, b^{\prime}, c^{\prime}>0$. Let $i, j, k \in[d]$ be such that $u, v, w$ lie in $T_{i}, T_{j}, T_{k}$, respectively. Attach intervals of lengths $a^{\prime}, b^{\prime}, c^{\prime}$ to $T$ at $u^{\prime}, v^{\prime}, w^{\prime}$, respectively, and call the resulting tree $T^{\prime}$. Let $u^{\prime \prime}, v^{\prime \prime}, w^{\prime \prime} \in T^{\prime}$ be the boundary points of those intervals. Then one obtains a new gluing datum $\left(T^{\prime}, d+1, \sim^{\prime}\right)$ from $(T, d, \sim)$ by gluing $T_{d+1}^{\prime}$ only along the points $u^{\prime \prime}, v^{\prime \prime}, w^{\prime \prime}$ with $T_{i}, T_{j}, T_{k}$, respectively, and leaving the gluing relation on the other copies of $T^{\prime}$ unchanged. Let $\Gamma^{\prime}$ be the corresponding metric graph. The following is now straightforward.

Lemma 15. The graph $\Gamma^{\prime}$ is a modification of the graph obtained from $\Gamma$ by adding a new trivalent vertex with new edges attached to $u, v, w$ of lengths $a+2 a^{\prime}, b+2 b^{\prime}, c+$ $2 c^{\prime}$, respectively. 
As an example, in the figure below, the graph $\Gamma$ together with its tropical morphism $\varphi: \Gamma \rightarrow T$ is pictured on the left. The right hand side shows the tropical morphism $\varphi^{\prime}: \Gamma^{\prime} \rightarrow T^{\prime}$, where the top tree is attached to $\Gamma$, and next to it, the graph $\Gamma^{\prime}$ with the dangling trees removed.
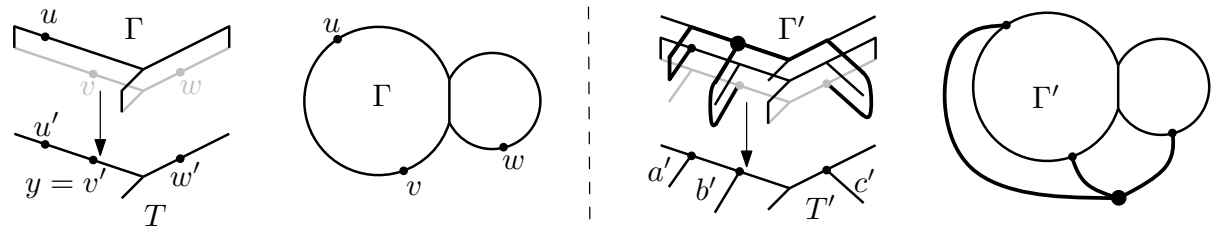

\section{A lower bound on the dimension in Theorem 1 .}

Proof of Theorem 1, lower bound. We proceed by induction on $d \geq 2$ to exhibit a subset $\mathcal{S}_{g, d}$ of $\mathcal{M}_{g}^{\text {trop }}$ of dimension $e(g, d)=\min \left\{2 g+2 d-5, \operatorname{dim} \mathcal{M}_{g}^{\text {trop }}\right\}$ where the gonality is at most $d$. The subset will be presented by a finite graph $G=(V, E)$ all of whose vertices are trivalent, together with an open polyhedral cone $U$ of dimension $e$ in $\mathbb{R}_{>0}^{E}$ consisting of edge lengths for which the gonality is at most $d$.

For $d=2$ we are concerned with the locus in $\mathcal{M}_{g}^{\text {trop }}$ of hyperelliptic metric graphs. This locus is well-understood Cha13, and its dimension is $2 g-1=e(g, 2)$ for all $g \geq 1$. Here is a gluing datum witnessing this dimension:
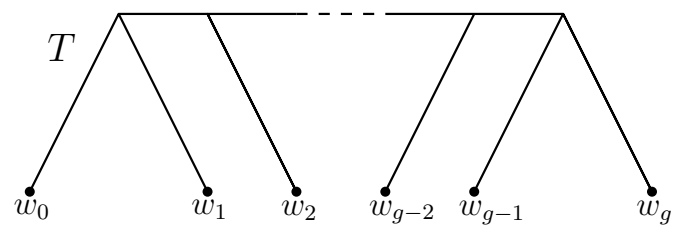

$$
\begin{aligned}
& \sim \text { on } S=T_{1} \sqcup T_{2} \\
& w_{i} \in T_{1} \sim w_{i} \in T_{2}
\end{aligned}
$$

Next let $d \geq 3$ and assume that we have found suitable subsets $\mathcal{S}_{g, d-1}$ for all $g$. Then to find $\mathcal{S}_{g, d}$ we pick a suitable subset $\mathcal{S}_{g-2, d-1}$, representated by $(G, U)$. Note that $e(g, d)=e(g-2, d-1)+6$ provided that $g-2$ is at least 2 , since then both of the terms in the minimum increase by 6 . If $g-2 \geq 2$, then any metric graph of genus $g-2$ has only finitely many automorphisms. Consequently, the number of degrees of additional freedom when gluing in a tripod is also 6: this counts the positions of $u, v, w$ and the positive numbers $a^{\prime}, b^{\prime}, c^{\prime}$, and the count does not drop modulo finitely many automorphisms. Hence we are done for $g \geq 4$.

We deal with the cases $g=2,3$ separately. Every graph with $g=2$ is hyperelliptic, so for $\mathcal{S}_{g, d}$ with $d \geq 2$ we can take any of the cells defining $\mathcal{M}_{2}^{\text {trop }}$. This leaves the case where $g=3$ and $d=3$. Here $e(3,3)=6$, and we obtain a subset $\mathcal{S}_{3,3}$ by gluing in a tripod in a cycle - the reason that this raises the dimension by 5 rather than 6 is that the automorphism group of the cycle can move one of the points, say $u$, to any fixed position.

Realising all combinatorial types. We now have almost all ingredients for proving Theorem 2, but one more notion is needed for the existence of a rank-one divisor supported at integral vertices. A finite subset $S$ of a metric graph $\Gamma$ is called an integral set if $\Gamma \backslash S$ is a union of open intervals of length 1 and half-open intervals of length strictly smaller than 1 . The metric graph $\Gamma$ has an integral set if and only if it is either a line segment of arbitrary length, or a single cycle of integral length, or it has at least one vertex of valency at least three and every line segment 
connecting two such vertices has integral length. In the last case, the integral set is unique, and the closed ends of the half-open intervals are necessarily valency-one vertices of $\Gamma$.

Proof of Theorem 2. We proceed by induction on $g$ to construct an open cone $C_{G} \subseteq$ $\mathbb{R}_{>0}^{E}$ for each trivalent graph $G$ of genus $g$ such that graphs $\Gamma$ represented by points in $C_{G}$ have gonality at most $\lceil(g+2) / 2\rceil$. Moreover, we will do this in such a way that each metric graph $\Gamma$ corresponding to a point in $\mathbb{Z}^{E} \cap C_{G}$ has a modification $\Gamma^{\prime}$ that admits a tropical morphism $\varphi: \Gamma^{\prime} \rightarrow T$ of degree $\lceil(g+2) / 2\rceil$ to a tree $T$ with the following additional properties:

(1) $\varphi$ maps some integral set $S^{\prime} \subseteq \Gamma^{\prime}$ into some integral set in $T$;

(2) $S:=S^{\prime} \cap \Gamma$ is an integral set in $\Gamma$; and

(3) there exists a point $v_{0} \in S$ such that $\varphi^{-1}\left(\varphi\left(v_{0}\right)\right) \cap \Gamma \subseteq S$.

Then the divisor $\sum_{v^{\prime} \in \varphi^{-1}\left(\varphi\left(v_{0}\right)\right)} m_{\varphi}\left(v^{\prime}\right) v^{\prime}$ on $\Gamma^{\prime}$ has rank one, and this remains the case if we move the chips on $\Gamma^{\prime} \backslash \Gamma$ to their nearest point on $\Gamma$; since the points where trees are grafted onto $\Gamma$ to obtain $\Gamma^{\prime}$ are necessarily in the integral set $S$, this latter divisor is supported on $S$.

For $g=1$ it is not quite clear how even to define a graph of genus 1 in which all vertices have valency 3 , but we take as definition the circle with no vertices. For this graph the statement is clear: for any length $a>0$ prescribed to the circle, it has a $2: 1$-morphism $\varphi$ to an interval of length $a / 2$. If $a$ is integral, then after choosing an integral set $S$ in the cycle, we can choose $\varphi$ such that $\varphi^{-1}(\varphi(S))=S$.

For $g=2$ there are two possible combinatorial types, and the statements to be proved are well known for both (the marked points $w$ on $T$ have equivalence class $\{1,2\}$ for $\left.\sim_{w}\right)$ :
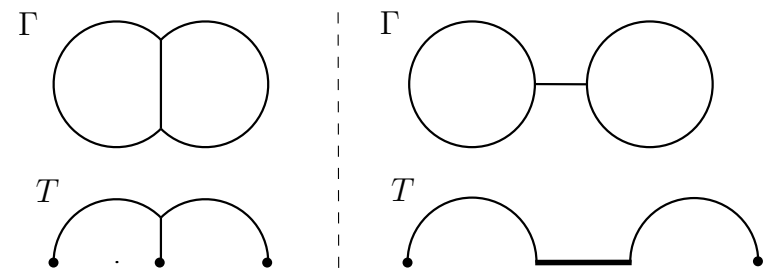

In each of the two cases, the open cone equals $\mathbb{R}_{>0}^{E}$, and also the integrality statements are readily verified.

Next, assume that $G=(V, E)$ is a trivalent graph of genus $g>2$. If $G$ has a vertex $y$ that can be removed without disconnecting the graph, then let $G^{\prime}=$ $\left(V^{\prime}, E^{\prime}\right)$ be the graph obtained by removing $y$ and its three incident edges $e_{1}, e_{2}, e_{3}$. Note that $G^{\prime}$ has genus $g-2$. By assumption, there is an open cone $C_{G^{\prime}} \subseteq \mathbb{R}_{>0}^{E^{\prime}}$ of dimension $\operatorname{dim} \mathcal{M}_{g-2}^{\text {trop }}$ consisting of edge lengths leading to metric graphs with gonality at most $\left\lceil\frac{g}{2}\right\rceil$. By gluing in a tripod we find an open cone $C_{G} \subseteq \mathbb{R}^{E}$ of the right dimension where the gonality is at most $1+\left\lceil\frac{g}{2}\right\rceil=\left\lceil\frac{g+2}{2}\right\rceil$ : the inequalities for $C_{G}$ are those for $C_{G^{\prime}}$ plus the conditions that the lengths of $e_{1}, e_{2}, e_{3}$ are sufficiently large (in the terminology of the subsection on gluing a tripod: larger than $a, b, c$, respectively).

To see that the integrality conditions are preserved, let $\Gamma$ be the metric graph corresponding to a point in $C_{G} \cap \mathbb{Z}^{E}$. The restriction to $E^{\prime}$ defines a metric graph 
$\Sigma$ of combinatorial type $G^{\prime}$, and by the induction hypothesis there is tropical morphism $\psi$ from a modification $\Sigma^{\prime}$ of $\Sigma$ to a tree $K$ of degree $\left\lceil\frac{g}{2}\right\rceil$ that satisfies the integrality conditions: $\Sigma^{\prime}$ has an integral set $R^{\prime}$ such that $\psi\left(R^{\prime}\right)$ is contained in an integral set $U$ of $K, R:=\Sigma \cap R^{\prime}$ is an integral set in $\Sigma$, and $v_{0} \in R$ is such that $\psi^{-1}\left(\psi\left(v_{0}\right)\right) \cap \Sigma \subseteq R$.

Let $\varphi$ be the tropical morphism $\Gamma^{\prime} \rightarrow T$ obtained via gluing a tripod to the points $u, v, w \in \Sigma$ of $e_{1}, e_{2}, e_{3}$ with edge lengths $a+2 a^{\prime}, b+2 b^{\prime}, c+2 c^{\prime}$ as in the subsection on tripods, and with central vertex $y$. Here $\Gamma^{\prime}$ is a modification of $\Gamma$. Then $u, v, w \in R$, and hence $\psi(u), \psi(v), \psi(w) \in U$. We extend $U$ to an integral set $V$ of $T$ by adding the vertices on the new edges (of lengths $a^{\prime}, b^{\prime}, c^{\prime} \in \frac{1}{2} \mathbb{Z}$ ) at an integral distance from $\psi(u), \psi(v), \psi(w)$, respectively. Next, we extend $R^{\prime}$ to an integral set $S^{\prime}$ of $\Gamma^{\prime}$ by

$$
S^{\prime}:=R^{\prime} \cup\left\{v \in \Gamma^{\prime} \backslash \Sigma^{\prime} \mid \varphi(v) \in V\right\} .
$$

Set $S:=S^{\prime} \cap \Gamma$. Then we find that each $x \in \varphi^{-1}\left(\varphi\left(v_{0}\right)\right) \cap \Gamma$ is either in $\psi^{-1}\left(\psi\left(v_{0}\right)\right) \cap$ $\Sigma \subseteq R \subseteq S$, or else in $\Gamma^{\prime} \backslash \Sigma^{\prime}$ and hence, since $\varphi(x)=\varphi\left(v_{0}\right) \in U$, also $x \in S$. Thus $S^{\prime}$ has the required property.

This concludes the proof for the case where $G$ has a trivalent vertex $y$ such that removing $y$ does not disconnect the graph. If, on the other hand, removing any vertex $y$ of $G$ disconnects $G$, then any two distinct simple cycles in $G$ intersect in at most one vertex, i.e., $G$ is a cactus graph. This case is dealt with by the proposition below.

Example 16. In the following example, we see two metric graphs of genera 2 and 4 , respectively, on the left, along with tropical morphisms of degrees 2 and 3 , as constructed above:
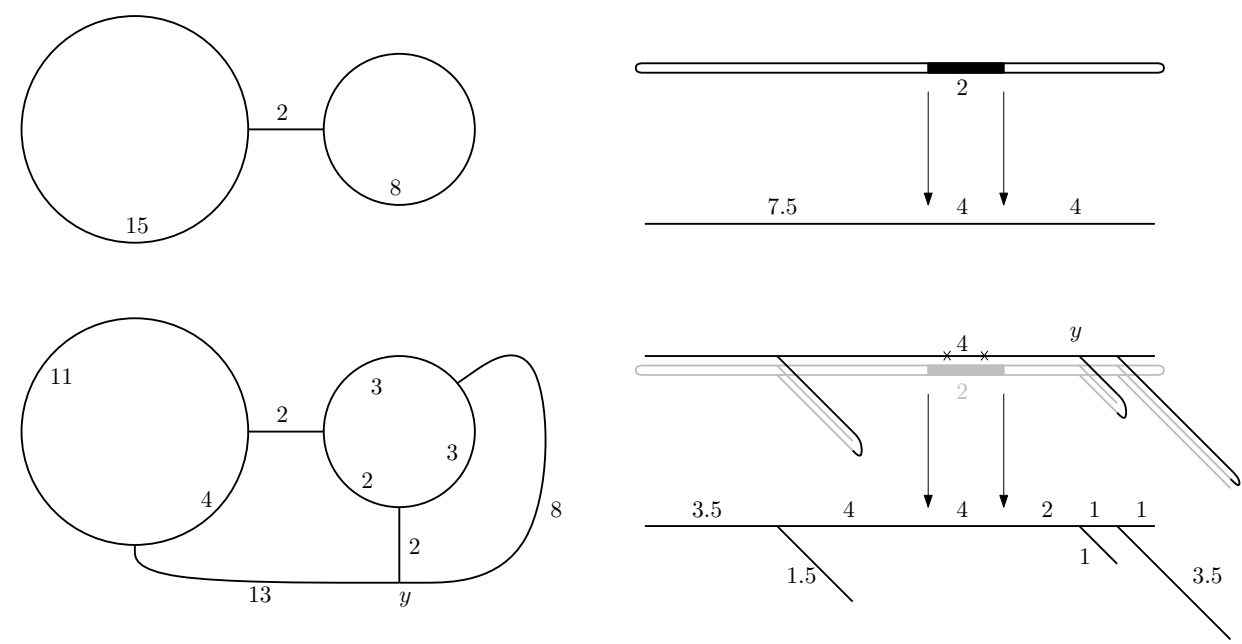

Focussing on the latter morphism $\varphi$, note that the segment between the two arrows has length 4 in the tree, and also in one of the copies of the tree above, but length 2 where the other two copies are glued together. The two marked points are in the

\footnotetext{
${ }^{1}$ Actually, there is one case where this is not automatic, namely, when $\Sigma$ is a single cycle. But in this case we can take $\Sigma^{\prime}$ equal to $\Sigma$ and choose $R^{\prime}$ such that it contains $u, v, w$.
} 
graph's integral set, but have a strictly half-integral point in their fibre. So they would not be a valid choice for $v_{0}$ in condition (3) above.

Cactus graphs. A metric graph $\Gamma$ is called a cactus graph if any two simple cycles (i.e., injective, continuous images of $S^{1}$ ) intersect in at most one point.

Proposition 17. Any metric cactus graph $\Gamma$ has a modification $\Gamma^{\prime}$ with a tropical morphism $\varphi$ from $\Gamma^{\prime}$ to a tree $T$, of degree $\left\lceil\frac{g(\Gamma)+2}{2}\right\rceil$, with the following additional constraints:

(1) if $g(\Gamma)$ is odd and $v_{1} \in \Gamma$ is any point, then $\varphi$ can be chosen such that $m_{\varphi}\left(v_{1}\right)=2$ and that moreover $k \geq 2 l-1$ where $k$ is the valency of $v_{1}$ in $\Gamma$ and $l$ is the valency of $\varphi\left(v_{1}\right)$ in $T$;

(2) if $\Gamma$ has an integral set $S$ (containing $v_{1}$ if $g(\Gamma)$ is odd), then $\Gamma^{\prime}$ has an integral set $S^{\prime}$ containing $S, \varphi\left(S^{\prime}\right)$ is contained in an integral set of $T$, and $\varphi^{-1}\left(\varphi\left(S^{\prime}\right)\right) \subseteq S^{\prime}$.

The former condition implies that the Riemann-Hurwitz inequality $k-2 \geq$ $2(l-2)$ holds at $v_{1}$ with a strict inequality. The latter condition is stronger than condition (3) in the proof above, where we require only that the fibre through some integral point intersects $\Gamma$ only inside $S$. Example 16 below shows why we could not impose this stronger condition earlier.

Proof. We proceed by induction on $g$. For $g=0$ we take $T=\Gamma^{\prime}=\Gamma$ and $\varphi$ the identity map. For $g=1$ let $C$ be the unique simple cycle in $\Gamma$, of length $a>0$, and let $w$ be the point on $C$ closest to the prescribed point $v_{1}$. The $2: 1$ map from $C$ to an edge with branchpoints $w$ and the point at distance $a / 2$ from $w$ extends to a modification of $\Gamma$ that has slope 1 everywhere except for slope 2 on the segment connecting $v_{1}$ and $w$ :
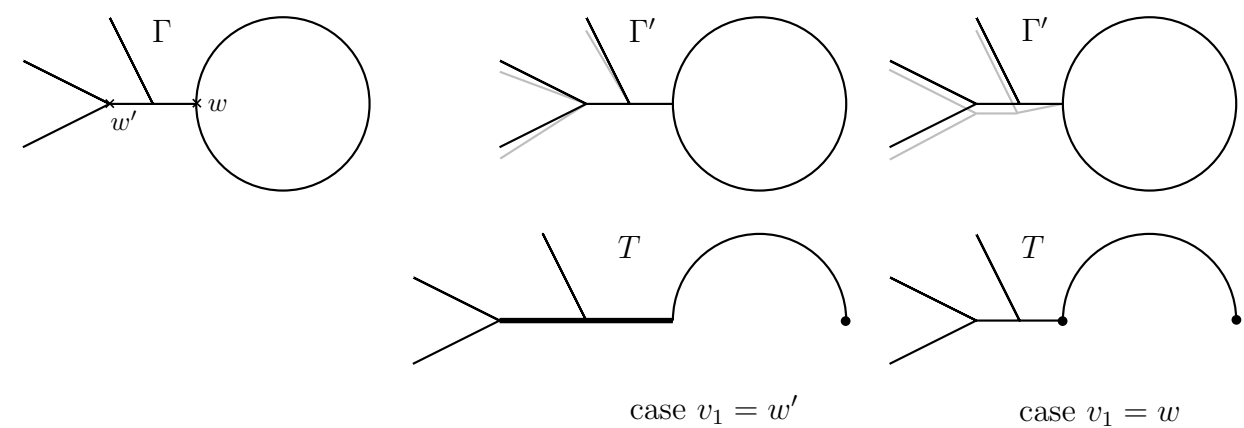

For (1) we note that $k$ is at least $2 l-1$, as required. For the integrality condition (2), we note that any integral set $S$ of $\Gamma$ has a unique extension to an integral set $S^{\prime}$ of $\Gamma^{\prime}$, and that there is a unique integral set in $T$ containing the image of $S^{\prime}$. Note that the latter inclusion is strict if the segment from $v_{1}$ to $w$ has positive (integral) length. Yet, $\varphi^{-1}(\varphi(S))=S$, as required.

If $\Gamma$ has higher genus, then we can write it as $\Gamma=\Gamma_{1} \cup \Gamma_{2}$ where $\Gamma_{1}, \Gamma_{2} \subseteq \Gamma$ are cactus graphs of lower genus than $\Gamma$ that intersect in a single point $y$ of $\Gamma$, which we can chose integral if $\Gamma$ has an integral set. Write $g:=g(\Gamma)$ and $g_{i}:=g\left(\Gamma_{i}\right)$ for $i=1,2$, and note that $g=g_{1}+g_{2}$. Moreover, if $g$ is odd, then we can (and do) choose the decomposition such that $g_{1}$ is odd and that the prescribed point $v_{1}$ lies 
in $\Gamma_{1}$. Furthermore, if $\Gamma$ has an integral set $S$, then $S \cap \Gamma_{i}$ is an integral set for $\Gamma_{i}$ for each $i=1,2$.

By the induction hypothesis, there are modifications $\Gamma_{i}^{\prime}, i=1,2$ of the $\Gamma_{i}$ with tropical morphisms $\varphi_{i}$ of degrees $\left\lceil g_{i} / 2\right\rceil+1$ to trees $T_{i}$ which further satisfy conditions (1) and (2) in the proposition. Here we choose $v_{1}$ equal to $y$ for both $\varphi_{1}$ and $\varphi_{2}$ if both $g_{1}$ and $g_{2}$ are odd (in this case, since $g$ is even, no point $v_{1}$ had yet been prescribed).

Let $T$ be the tree obtained by gluing $T_{1}$ and $T_{2}$ at $\varphi_{1}(y)$ and $\varphi_{2}(y)$, respectively, and let $\Gamma^{\prime}$ be the metric graph obtained by gluing $\Gamma_{1}^{\prime}, \Gamma_{2}^{\prime}$ at $y$. The metric graph $\Gamma^{\prime}$ is a modification of $\Gamma$, and an integral set of $\Gamma$ extends uniquely to one of $\Gamma^{\prime}$. Let $\psi: \Gamma^{\prime} \rightarrow T$ be the map restricting to $\varphi_{i}$ on $\Gamma_{i}^{\prime}$. Then $\psi$ is harmonic except in the points in $Y:=\psi^{-1}(\psi(y))$. Apart from $y$, which belongs to both $\Gamma_{i}^{\prime}$, the points in $Y$ are either in $\Gamma_{1}^{\prime}$ or in $\Gamma_{2}^{\prime}$ but not both. Let $v_{0}:=y, v_{1}, \ldots, v_{a}$ be the points in $Y \cap \Gamma_{1}^{\prime}$ and let $w_{0}:=y, w_{1}, \ldots, w_{b}$ be the points in $Y \cap \Gamma_{2}^{\prime}$, and write $m_{i}:=m_{\varphi_{1}}\left(v_{i}\right)$ for $i=0, \ldots, a$ and $n_{j}:=m_{\varphi_{2}}\left(w_{j}\right)$ for $j=0, \ldots, b$. We modify $\Gamma^{\prime}$ by grafting $m_{i}$ copies of $T_{2}$ at $v_{i}$ for $i=1, \ldots, a$ (not yet at $v_{0}=y$ !) and grafting $n_{j}$ copies of $T_{1}$ at $w_{j}$ for $j=1, \ldots, b$ (not yet at $w_{0}=y !$ ), and we extend $\psi$ to these copies by their natural maps into $T$. This renders $\psi$ harmonic at $v_{1}, \ldots, v_{a}$ and $w_{1}, \ldots, w_{b}$, and moreover restores the Riemann-Hurwitz condition there-e.g., to the valency $v_{i}$ one adds $m_{i}$ times the valency of $\varphi_{2}(y)$ in $T_{2}$, which is exactly $m_{i}$ times what was added to the valency of $\varphi_{1}\left(v_{i}\right)=\varphi(y)$ by attaching $T_{2}$.

So we need only establish harmonicity and the Riemann-Hurwitz condition at $y$. Let $d_{i}=\left\lceil g_{i} / 2\right\rceil+1$ be the degree of $\varphi_{i}$. First, assume that $g_{2}$ is even. Then the required degree of the map $\varphi$ equals

$$
d:=\lceil g / 2\rceil+1=\left\lceil g_{1} / 2\right\rceil+g_{2} / 2+1=d_{1}+d_{2}-1 .
$$

We graft $m_{0}-1$ further copies of $T_{2}$ and $n_{0}-1$ copies of $T_{1}$ to $y$; this yields the final modification $\Gamma^{\prime \prime}$ of $\Gamma$ with the natural extension $\varphi: \Gamma^{\prime \prime} \rightarrow T$ of $\psi$. This extension is harmonic everywhere by construction. To check the Riemann-Hurwitz condition at $y$, let $k_{i}, k$ denote the valency of $y$ in $\Gamma_{i}^{\prime}$ and $\Gamma^{\prime \prime}$, respectively, and let $l_{i}, l$ denote the valency of $\varphi_{i}(y)$ and $\varphi(y)$ in $T_{i}, T$, respectively. Then we have $l=l_{1}+l_{2}$ and $k=k_{1}+k_{2}+\left(m_{0}-1\right) l_{2}+\left(n_{0}-1\right) l_{1}$, so that

$$
\begin{aligned}
k-2 & =k_{1}+k_{2}+\left(m_{0}-1\right) l_{2}+\left(n_{0}-1\right) l_{1}-2 \\
& \geq m_{0}\left(l_{1}-2\right)+2+n_{0}\left(l_{2}-2\right)+2+\left(m_{0}-1\right) l_{2}+\left(n_{0}-1\right) l_{1}-2 \\
& =\left(m_{0}+n_{0}-1\right)\left(l_{1}+l_{2}-2\right) \\
& =m_{\varphi}(y)(l-2),
\end{aligned}
$$

where the inequality follows from the Riemann-Hurwitz inequalities for the $\varphi_{i}$.

Second, assume that $g_{2}$ is odd; then, by assumption, so is $g_{1}$. With notation as above we now have

$$
d=\lceil g / 2\rceil+1=d_{1}+d_{2}-2 .
$$

Moreover, since we had chosen $y$ as the prescribed point for both $\varphi_{1}$ and $\varphi_{2}$, we have $m_{0}=n_{0}=2$ by property (1). This means that we need not graft further trees at $y$ and the map $\varphi: \Gamma^{\prime \prime} \rightarrow T$ constructed so far is harmonic there. To check the Riemann-Hurwitz conditions, we compute

$$
k-2=k_{1}+k_{2}-2 \geq 2 l_{1}-1+2 l_{2}-1-2=2(l-2)=m_{\varphi}(y)(l-2),
$$

where we have used that $k_{i} \geq 2 l_{i}-1$ by property $(1)$. 
Finally, if $\Gamma$ has an integral set $S$, then $\Gamma^{\prime \prime}$ has a unique integral set $S^{\prime}$ containing $S$, and it contains the integral sets $S_{i}^{\prime}=S^{\prime} \cap \Gamma_{i}^{\prime}$ as well as suitable integral sets of the trees grafted onto $\Gamma^{\prime}$ to arrive at $\Gamma^{\prime \prime}$. The points in $\varphi^{-1}\left(\varphi\left(S^{\prime}\right)\right)$ that are not in the union of the sets $\varphi_{i}^{-1}\left(\varphi_{i}\left(S_{i}^{\prime}\right)\right)$ are in those integral sets of the grafted trees, hence also in $S^{\prime}$.

Remark 18. (1) Combining this subsection with the previous one, we find that metric graphs in the open cone $C_{G}$ have a modification with a tropical morphism to a tree that only has slopes 1 and 2 .

(2) The existence of divisors of higher rank on cactus graphs, under the condition that the Brill-Noether number is nonnegative, was studied by Jorn van der Pol in his Bachelor's thesis vdP11] under an additional assumption on the cactus graph.

(3) The existence of integral rank-one divisors of degree $\lceil(g+2) / 2\rceil$ on an arbitrary graph of genus $g$ remains conjectural. Conceivably, Backman's approach to linear equivalence using graph orientations [Bac17] could lead to such a result. In any case, following our approach in this paper, we do not see how to cross the boundary of the cone $C_{G}$.

\section{ACKNOWLEDGMEnTs}

We thank Aart Blokhuis, who provided the proof of Lemma 9 reproduced in this paper. JD was partially supported by Vidi and Vici grants from the Netherlands Organisation for Scientific Research (NWO).

\section{REFERENCES}

[AB15] Omid Amini and Matthew Baker. Linear series on metrized complexes of algebraic curves. Math. Ann., 362(1-2):55-106, 2015.

[ABBR15a] Omid Amini, Matthew Baker, Erwan Brugallé, and Joseph Rabinoff. Lifting harmonic morphisms I: metrized complexes and Berkovich skeleta. Res. Math. Sci., 2:Art. 7, $67,2015$.

[ABBR15b] Omid Amini, Matthew Baker, Erwan Brugallé, and Joseph Rabinoff. Lifting harmonic morphisms II: Tropical curves and metrized complexes. Algebra Number Theory, 9(2):267-315, 2015.

[ACP15] Dan Abramovich, Lucia Caporaso, and Sam Payne. The tropicalization of the moduli space of curves. (La tropicalisation de lespace des modules des courbes.). Ann. Sci. Éc. Norm. Supér. (4), 48(4):765-809, 2015.

[AK16] Omid Amini and Janne Kool. A spectral lower bound for the divisorial gonality of metric graphs. Int. Math. Res. Not. IMRN, 8:2423-2450, 2016.

[Bac17] Spencer Backman. Riemann-Roch theory for graph orientations. Adv. Math., 309:655691, 2017.

[Bak08] Matthew Baker. Specialization of linear systems from curves to graphs (with an appendix by Brian Conrad). Algebra Number Theory, 2(6):613-653, 2008.

[BBM11] Benoît Bertrand, Erwan Brugallé, and Grigory Mikhalkin. Tropical open Hurwitz numbers. Rend. Semin. Mat. Univ. Padova, 125:157-171, 2011.

[BBM14] Benoît Bertrand, Erwan Brugallé, and Grigory Mikhalkin. Genus 0 characteristic numbers of the tropical projective plane. Compos. Math., 150(1):46-104, 2014.

[BN07] Matthew Baker and Serguei Norine. Riemann-Roch and Abel-Jacobi theory on a finite graph. Adv. Math., 215(2):766-788, 2007.

[BN09] Matthew Baker and Serguei Norine. Harmonic morphisms and hyperelliptic graphs. Int. Math. Res. Not., 2009(15):2914-2955, 2009.

[Cap12] Lucia Caporaso. Geometry of tropical moduli spaces and linkage of graphs. J. Comb. Theory, Ser. A, 119(3):579-598, 2012. 
[Cap14] Lucia Caporaso. Gonality of algebraic curves and graphs. In Algebraic and complex geometry. In honour of Klaus Hulek's 60th birthday. Based on the conference on algebraic and complex geometry, Hannover, Germany, September 10-14, 2012, pages 77-108. Cham: Springer, 2014.

[CDPR12] Filip Cools, Jan Draisma, Sam Payne, and Eline Robeva. A tropical proof of the Brill-Noether Theorem. Adv. Math, 230(2):759-776, 2012.

[Cha13] Melody Chan. Tropical hyperelliptic curves. J. Algebraic Combin., 37(2):331-359, 2013.

[CKK15] Gunther Cornelissen, Fumiharu Kato, and Janne Kool. A combinatorial Li-Yau inequality and rational points on curves. Math. Ann., 361(1-2):211-258, 2015.

[DV17] Jan Draisma and Alejandro Vargas. Catalan-many tropical morphisms to trees; or, Chip-firing in the space of graphs. 2017. Preprint.

[HKN13] Jan Hladký, Daniel Král, and Serguei Norine. Rank of divisors on tropical curves. J. Combin. Theory Ser. A, 120(7):1521-1538, 2013.

[Kem71] G. Kempf. Schubert methods with an application to algebraic curves. Math. Centrum, Amsterdam, Afd. zuivere Wisk. ZW 6/71, 18 p. (1971)., 1971.

[KL72] Steven L. Kleiman and Dan Laksov. On the existence of special divisors. Am. J. Math., 94:431-436, 1972.

[Koz09] Dmitry N. Kozlov. The topology of moduli spaces of tropical curves with marked points. Asian J. Math., 13(3):385-404, 2009.

[LM14] Ye Luo and Madhusudan Manjunath. Smoothing of limit linear series of rank one on saturated metrized complexes of algebraic curves. Preprint, arxiv:1411.2325, 2014.

[Luo11] Ye Luo. Rank-determining sets of metric graphs. J. Comb. Theory, Ser. A, 118(6):1775-1793, 2011.

[Mik11] Grigory Mikhalkin. Regularity, realizability and Brill-Noether theory for tropical curves, 2011. Talk at the Conference on tropical geometry and computational biology, Saarbrücken, September 26-30, 2011, www.math.uni-sb.de/wiki/doku.php?id=ag-seite:ag-markwig: conferences: tropbio.

[Seg28] B. Segre. Sui moduli delle curve poligonale, e sopra un complemento al teorema di esistenza di Riemann. Math. Ann., 100:537-551, 1928.

[vDdBG14] Josse van Dobben de Bruyn and Dion Gijswijt. Treewidth is a lower bound on graph gonality. Preprint, arxiv:1407.7055, 2014.

[vdP11] Jorn G. van der Pol. Analysis of the brill-noether game on metric cactus graphs. Bachelor report, 2011. available online from repository.tue.nl/.

(Filip Cools) Department of Mathematics, Katholieke Universiteit Leuven, CelestijNENLAAN 200B - BOX 2400, 3001 LeUven, Belgium

E-mail address: filip.cools@wis.kuleuven.be

(Jan Draisma) Department of Mathematics and Computer Science, Technische Universiteit Eindhoven, P.O. Box 513, 5600 MB Eindhoven, The Netherlands; and Vrije Universiteit Amsterdam, The Netherlands

E-mail address: j.draisma@tue.nl 